\title{
Mitigating Gender Bias in Natural Language Processing: Literature Review
}

\author{
Tony Sun ${ }^{* \dagger}$, Andrew Gaut ${ }^{\dagger}$, Shirlyn Tang ${ }^{\dagger}$, Yuxin Huang ${ }^{\dagger}$, \\ Mai ElSherief ${ }^{\dagger}$, Jieyu Zhao ${ }^{\ddagger}$, \\ Diba Mirza $^{\dagger}$, Elizabeth Belding ${ }^{\dagger}$, Kai-Wei Chang ${ }^{\ddagger}$, and William Yang Wang ${ }^{\dagger}$ \\ ${ }^{\dagger}$ Department of Computer Science, UC Santa Barbara \\ ${ }^{\ddagger}$ Department of Computer Science, UC Los Angeles \\ \{tonysun, ajg, shirlyntang, yuxinhuang\}@ucsb.edu \\ \{mayelsherif, dimirza, ebelding, william\}@cs.ucsb.edu \\ \{jyzhao, kwchang\}@es.ucla.edu
}

\begin{abstract}
As Natural Language Processing (NLP) and Machine Learning (ML) tools rise in popularity, it becomes increasingly vital to recognize the role they play in shaping societal biases and stereotypes. Although NLP models have shown success in modeling various applications, they propagate and may even amplify gender bias found in text corpora. While the study of bias in artificial intelligence is not new, methods to mitigate gender bias in NLP are relatively nascent. In this paper, we review contemporary studies on recognizing and mitigating gender bias in NLP. We discuss gender bias based on four forms of representation bias and analyze methods recognizing gender bias. Furthermore, we discuss the advantages and drawbacks of existing gender debiasing methods. Finally, we discuss future studies for recognizing and mitigating gender bias in NLP.
\end{abstract}

\section{Introduction}

Gender bias is the preference or prejudice toward one gender over the other (Moss-Racusin et al., 2012). Gender bias is exhibited in multiple parts of a Natural Language Processing (NLP) system, including the training data, resources, pretrained models (e.g. word embeddings), and algorithms themselves (Zhao et al., 2018a; Bolukbasi et al., 2016; Caliskan et al., 2017; Garg et al., 2018). NLP systems containing bias in any of these parts can produce gender biased predictions and sometimes even amplify biases present in the training sets (Zhao et al., 2017).

The propagation of gender bias in NLP algorithms poses the danger of reinforcing damaging

\footnotetext{
* Equal Contribution.
}

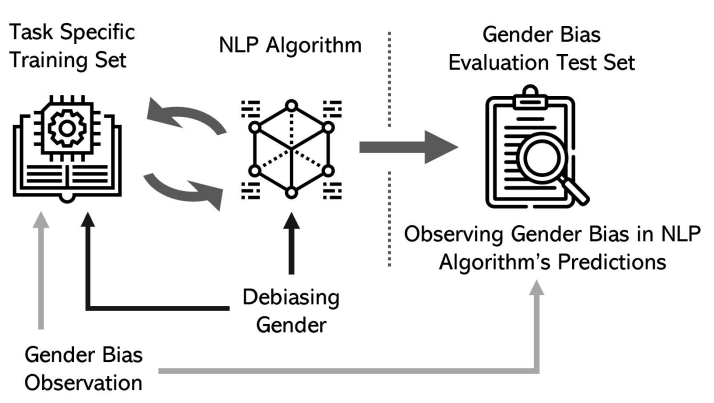

Figure 1: Observation and evaluation of gender bias in NLP. Bias observation occurs in both the training sets and the test sets specifically for evaluating the gender bias of a given algorithm's predictions. Debiasing gender occurs in both the training set and within the algorithm itself.

stereotypes in downstream applications. This has real-world consequences; for example, concerns have been raised about automatic resume filtering systems giving preference to male applicants when the only distinguishing factor is the applicants' gender.

One way to categorize bias is in terms of allocation and representation bias (Crawford, 2017). Allocation bias can be framed as an economic issue in which a system unfairly allocates resources to certain groups over others, while representation bias occurs when systems detract from the social identity and representation of certain groups (Crawford, 2017). In terms of NLP applications, allocation bias is reflected when models often perform better on data associated with majority gender, and representation bias is reflected when associations between gender with certain concepts are captured in word embedding and model parameters. In Table 1, we categorize common examples of gender bias in NLP following Crawford (2017). 


\begin{tabular}{|l|l|c|c|c|c|}
\hline Task & Example of Representation Bias in the Context of Gender & D & S & R & U \\
\hline $\begin{array}{l}\text { Machine } \\
\text { Translation }\end{array}$ & $\begin{array}{l}\text { Translating "He is a nurse. She is a doctor." to Hungarian and back to } \\
\text { English results in "She is a nurse. He is a doctor." (Douglas, 2017) }\end{array}$ & $\checkmark$ & $\checkmark$ & \\
\hline Caption Generation & $\begin{array}{l}\text { An image captioning model incorrectly predicts the agent to be male } \\
\text { because there is a computer nearby (Burns et al., 2018). }\end{array}$ & $\checkmark$ & $\checkmark$ & \\
\hline $\begin{array}{l}\text { Speech } \\
\text { Recognition }\end{array}$ & $\begin{array}{l}\text { Automatic speech detection works better with male voices than female } \\
\text { voices (Tatman, 2017). }\end{array}$ & & $\checkmark$ & $\checkmark$ \\
\hline Sentiment Analysis & $\begin{array}{l}\text { Sentiment Analysis Systems rank sentences containing female noun } \\
\text { phrases to be indicative of anger more often than sentences containing } \\
\text { male noun phrases (Park et al., 2018). }\end{array}$ & $\checkmark$ & & \\
\hline Language Model & $\begin{array}{l}\text { "He is doctor" has a higher conditional likelihood than "She is doctor" } \\
\text { (Lu et al., 2018). }\end{array}$ & $\checkmark$ & $\checkmark$ & $\checkmark$ \\
\hline Word Embedding & $\begin{array}{l}\text { Analogies such as "man : woman :: computer programmer : homemaker" } \\
\text { are automatically generated by models trained on biased word } \\
\text { embeddings (Bolukbasi et al., 2016). }\end{array}$ & $\checkmark$ & $\checkmark$ & $\checkmark$ & $\checkmark$ \\
\hline
\end{tabular}

Table 1: Following the talk by Crawford (2017), we categorize representation bias in NLP tasks into the following four categories: (D)enigration, (S)tereotyping, (R)ecognition, (U)nder-representation.

Briefly, denigration refers to the use of culturally or historically derogatory terms; stereotyping reinforces existing societal stereotypes; recognition bias involves a given algorithm's inaccuracy in recognition tasks; and under-representation bias is the disproportionately low representation of a specific group. We identify that both allocative and representational harms often arise in NLP systems due to statistical patterns in the training corpora, which are then embedded in semantic representations and the model.

Gender bias in NLP is a complex and compound issue, requiring interdisciplinary communication. As NLP systems have been increasingly integrated with our daily life thanks to modern AI developments, we need both immediate solutions to patch current systems as well as fundamental approaches to debias. In this paper, we provide a comprehensive literature review to summarize recent attempts for recognizing and mitigating bias in NLP systems. Most debiasing methods can be depicted as a special case in Figure 1.

We make two primary contributions. (1) We summarize recent studies of algorithmic bias in NLP under a unified framework for the ease of future discussion. (2) We critically discuss issues with current debiasing methods with the purpose of identifying optimizations, knowledge gaps, and directions for future research.

\section{Observing Gender Bias}

Recent work in analyzing gender bias in NLP has focused on quantifying bias through psychological tests, performance differences between genders for various tasks, and the geometry of vector spaces. We provide an overview of gender bias evaluation methods and discuss types of representation bias each method identifies.

\subsection{Adopting Psychological Tests}

In psychology, the Implicit Association Test (IAT) is used to measure subconscious gender bias in humans, which can be quantified as the difference in time and accuracy for humans to categorize words as relating to two concepts they find similar versus two concepts they find different (Greenwald et al., 1998; Caliskan et al., 2017). For instance, to measure subconscious associations of genders with arts and sciences, participants are asked to categorize words as pertaining to (males or the sciences) or (females or the arts) (Nosek et al., 2009). The participants are then asked to categorize words as pertaining to (males or the arts) or (females or the sciences). If participants answered faster and more accurately in the former setting, it indicates that humans subconsciously associate males with the sciences and females with the arts.

Caliskan et al. (2017) adopt the IAT's core concept, measuring gender bias through the difference in strength of association of concepts, to measure bias in word embeddings using the Word Embedding Association Test (WEAT) (Caliskan et al., 2017). The authors confirm that human biases found through IAT tests exist in GloVe and Word2Vec embeddings. Finally, the authors demonstrate a positive correlation between the strength of association of an occupation word embedding with the female gender and the percentage of females in that occupation in United States, with the percentages taken from Bureau of Labor Statistics labor force participation data. Notably, Garg et al. (2018) show that bias in word 
embeddings can be used to track social changes such as increased or decreased female participation in the workforce. May et al. (2019) extend WEAT to create the Sentence Encoder Association Test (SEAT), capable of testing sentence encoders (e.g., ELMo (Peters et al., 2018)) for human biases found in IAT tests.

\subsection{Analyzing Gender Sub-space in Embeddings}

Bolukbasi et al. (2016) define gender bias as the correlation between the magnitude of the projection onto the gender subspace of a word embedding representing a gender-neutral word and that word's bias rating, as rated by crowd workers. To identify the gender subspace, they first build a linear support vector machine to classify words into a set of gender-specific and a set of gender-neutral words based on a training set of hand-selected gender-specific words. The authors then identify a gender direction by aggregating ten gender pairs (e.g. she-he, her-his, woman-man, etc.) and using principal component analysis to find a single eigenvector that exhibits significantly greater variance than the rest. Manzini et al. (2019) extend this method and their approach can be used to find non-binary gender bias by aggregating n-tuples instead of gender pairs.

However, Gonen and Goldberg (2019) note that the above method fails to capture the full picture of gender bias in vector spaces. Specifically, even after the projections of word embeddings representing gender-neutral words onto the gender subspace have been removed, word embeddings representing words with similar biases still cluster together. They further introduce the notion of cluster bias. Cluster bias of a word $w$ can be measured as the percentage of male or female stereotypical words among the $k$ nearest neighbors of $w$ 's embedding where the male or female stereotypical words are obtained through human annotation.

\subsection{Measuring Performance Differences Across Genders}

In most NLP tasks, a model's prediction should not be heavily influenced by the gender of the entity mentions or contexts in the input. To evaluate whether or not this is the case, consider two sentences that act as the inputs to a model for which the only differences are the words that correspond to gender, such as "He went to the park" vs "She went to the park". We refer to changing the gen- der of the gendered nouns as gender-swapping. Gender-swapping can be generalized to sentences by swapping each male-definitional word with its respective female equivalent and vice-versa (Zhao et al., 2018a; Lu et al., 2018; Kiritchenko and Mohammad, 2018). If the model does not make decisions based on genders, it should perform equally for both sentences. Otherwise, the difference in evaluation scores reflects the extent of gender bias found in the system.

For example, Dixon et al. (2017) introduce two metrics to measure these performance differences - False Positive Equality Difference (FPED) and False Negative Equality Difference (FNED) - that have been used to measure gender bias in abusive language detection (Park et al., 2018). These are defined as the differences in the false positive and false negative rates, respectively, of predictions of a model between original and gender-swapped inputs. We note that these measurements can generalize to tasks aside from abusive language detection.

By designing test sets, measuring performance differences between genders reveals representational gender bias in the context of recognition, stereotyping, and under-representation. If, for instance, an image captioning model is worse at recognizing a woman than a man when they are each sitting in front of a computer (Burns et al., 2018), that is a clear indicator of recognition bias. If this prediction inaccuracy arises as a consequence of the algorithm's association between man and computer, then this example also reveals stereotyping in the image captioning model. One can also imagine that if the model is not debiased and these errors propagate over a large sample of images, then the model may further contribute to the under-representation of minority.

Standard evaluation data sets in NLP are inadequate for measuring gender bias. For one, these data sets often also contain biases (such as containing more male references than female references), so evaluation on them might not reveal gender bias. Furthermore, predictions made by systems performing complex NLP tasks depend on many factors; we must carefully design data sets to isolate the effect of gender of the output in order to be able to probe gender bias. We name these data sets Gender Bias Evaluation Testsets (GBETs).

The goal of designing GBETs is to provide 


\begin{tabular}{|l|l|l|l|}
\hline Data Set & Task & Probing Concept & Size \\
\hline Winogender Schemas (Rudinger et al., 2018) & Coreference Resolution & Occupation & 720 English Sentences \\
\hline WinoBias (Zhao et al., 2018a) & Coreference Resolution & Occupation & 3,160 English Sentences \\
\hline GAP (Webster et al., 2018) & Coreference Resolution & Names & 4,454 English Contexts \\
\hline EEC (Kiritchenko and Mohammad, 2018) & Sentiment Analysis & Emotion & 8,640 English Sentences \\
\hline
\end{tabular}

Table 2: Summary of GBETs. GBETs evaluate models trained for specific tasks for gender bias. GBETs use differences in values of the probing concept or prediction accuracies relating to the probing concept between gender-swapped data points to measure bias.

check that NLP systems avoid making mistakes due to gender bias. Some may argue that the artificial design of GBETs does not reflect the true distribution of the data, implying that these evaluations are artificial. We argue that if humans can avoid making mistakes due to gender bias, then machines should as well. Additionally, systems that make biased predictions may discourage minorities from using those systems and having their data collected, thus worsening the disparity in the data sets (Hashimoto et al., 2018). We provide an overview of publicly available GBETs in Table 2.

Gender-swapped GBETs: In the following, we review GBETs in coreference resolution and sentiment analysis applications.

For coreference resolution, Rudinger et al. (2018) and Zhao et al. (2018b) independently designed GBETs based on Winograd Schemas. The corpus consists of sentences which contain a gender-neutral occupation (e.g., doctor), a secondary participant (e.g., patient), and a gendered pronoun that refers either the occupation or the participant. The coreference resolution system requires the identification of the antecedent of the pronoun. For each sentence, Rudinger et al. (2018) consider three types of pronouns (female, male, or neutral), and Zhao et al. (2018b) consider male and female pronouns. The two datasets have a few notable differences (see the discussion in (Rudinger et al., 2018)).

Note that simply measuring a global difference in accuracies of a model between inputs with different gendered pronouns is insufficient. For example, a model could predict females and males to be coreferent to "secretary" with $60 \%$ and $20 \%$ accuracy, respectively. If that same model predicts females and males coreferent to "doctor" with $20 \%$ and $60 \%$ accuracy, respectively, then the global average accuracy for each gender is equivalent, yet the model exhibits bias. ${ }^{1}$ Therefore, Zhao

\footnotetext{
${ }^{1}$ For the sake of simplicity, we illustrate the motivation in accuracy. The coreference resolution systems may be evaluated using a different metric.
}

et al. (2018b) and Rudinger et al. (2018) design metrics to analyze gender bias by examining how the performance difference between genders with respect to each occupation correlate with the occupational gender statistics from the U.S Bureau of Labor Statistics.

Another GBET for coreference resolution named GAP contains sentences mined from Wikipedia and thus can perform an evaluation with sentences taken from real contexts as opposed to artificially generated ones (Webster et al., 2018). GAP does not include stereotypical nouns; instead, pronouns refer to names only. Gender bias can be measured as the ratio of $F_{1}$ scores on inputs for which the pronoun is female to inputs for which the pronoun is male. Notably, sentences are not gender-swapped, so there may be differences in difficulty between sentences in male and female test sets.

For sentiment analysis, a GBET dataset named Equity Evaluation Corpus (EEC) (Kiritchenko and Mohammad, 2018) is designed. Each EEC sentence contains an emotional word (e.g., anger, fear, joy, sadness), with one of five intensities for each emotion and a gender-specific word. Gender bias is measured as the difference in emotional intensity predictions between genderswapped sentences.

\section{Debiasing Methods Using Data Manipulation}

Several approaches have been proposed for debiasing gender stereotypes in NLP by working on two tangents: (1) text corpora and their representations and (2) prediction algorithms. In this section, we will discuss the techniques to debias text corpora and word embeddings. We do the same for techniques to mitigate gender bias in algorithms in Section 4.

We note that debiasing methods can be categorized as retraining and inference (see Table 3 ). Retraining methods require that the model is trained 
again, while inference methods reduce bias without requiring the existence of the original training set. Retraining methods tend to address gender bias in its early stages or even at its source. However, retraining a model on a new data set can be costly in terms of resources and time. Inference methods, on the other hand, do not require models to be retrained; instead, they patch existing models to adjust their outputs providing a testing-time debiasing. We will discuss different debiasing methods from these two perspectives.

\subsection{Debiasing Training Corpora}

We review three approaches for debiasing gender in the literature.

\subsubsection{Data Augmentation}

Oftentimes a data set has a disproportionate number of references to one gender (e.g. OntoNotes 5.0) (Zhao et al., 2018a). To mitigate this, Zhao et al. (2018a) proposed to create an augmented data set identical to the original data set but biased towards the opposite gender and to train on the union of the original and data-swapped sets. The augmented data set is created using gender-swapping. This is similar to the method used to create GBETs; however, the goal of data augmentation is to debias predictions by training the model on a gender-balanced data set, while GBETs are created specifically to evaluate the gender bias of those predictions both before and after debiasing.

Data augmentation works as follows: for every sentence in the original data set, create that sentence's gender-swapped equivalent using the procedure described in 2.3. Next, apply nameanonymization to every original sentence and its gender-swapped equivalent. Name anonymization consists of replacing all named entities with anonymized entities, such as "E1". For instance, Mary likes her mother Jan becomes El likes his father E2 after applying gender-swapping and name anonymization for data augmentation. This removes gender associations with named entities in sentences. The model is then trained on the union of the original data set with name-anonymization and the augmented data set. The identification of gender-specific words and their equivalent opposite gender word requires lists typically created by crowd workers.

Data augmentation has been shown to be flexible; it can mitigate gender bias in several differ-

\begin{tabular}{|l|l|}
\hline Methods & Method Type \\
\hline Data Augmentation by Gender-Swapping & Retraining \\
\hline Gender Tagging & Retraining \\
\hline Bias Fine-Tuning & Retraining \\
\hline Hard Debiasing & Inference \\
\hline Learning Gender-Neutral Embeddings & Retraining \\
\hline Constraining Predictions & Inference \\
\hline Adjusting Adversarial Discriminator & Retraining \\
\hline
\end{tabular}

Table 3: Debiasing methods can be categorized according to how they affect the model. Some debiasing methods require the model to be retrained after debiasing (Retraining). Others modify existing models' predictions or representations (Inference).

ent models in many different tasks. When applied to a neural network based coreference resolution model (Lee et al., 2017, 2018) originally trained on OntoNotes 5.0 which was tested on WinoBias, gender augmentation lowered the difference between $F_{1}$ scores on pro-stereotypical and antistereotypical test sets significantly, which indicates the model was less inclined to make genderbiased predictions (Zhao et al., 2018a, 2019). In hate speech detection, data augmentation reduced FNED and FPED differences between male and female predictions of a Convolutional Neural Network by a wide margin (Park et al., 2018). Data augmentation without name-anonymization has also been used to debias knowledge graphs built from Bollywood movie scripts (Madaan et al., 2018) by swapping the nodes for the lead actor and actress, but metrics evaluating the success of gender-swapping were not provided.

Data augmentation is easy to implement, but creating the annotated list can be expensive if there is high variability in the data or if the data set is large since more annotations will be required. Furthermore, data augmentation doubles the size of the training set, which can increase training time by a factor specific to the task at hand. Lastly, blindly gender-swapping can create nonsensical sentences - for example, gender-swapping "she gave birth" to "he gave birth" (Madaan et al., 2018).

\subsubsection{Gender Tagging}

In some tasks, like Machine Translation (MT), confounding the gender of the source of a data point can lead to inaccurate predictions. Current MT models predict the source to be male a disproportionate amount of time (Prates et al., 2018; Vanmassenhove et al., 2018). This happens because training sets are dominated by male-sourced 
data points, so the models learn skewed statistical relationships and are thus more likely to predict the speaker to be male when the gender of the source is ambiguous (Vanmassenhove et al., 2018).

Gender tagging mitigates this by adding a tag indicating the gender of the source of the data point to the beginning of every data point. For instance, "I'm happy" would change to "MALE I'm happy." In theory, encoding gender information in sentences could improve translations in which the gender of the speaker affects the translation (i.e. "I am happy" could translate to "Je suis heureux" $[\mathrm{M}]$ or "Je suis heureuse" [F]), since English does not mark the gender of the speaker in this case. The tag is then parsed separately from the rest of the data by the model. The goal is to preserve the gender of the source so the model can create more accurate translations (Vanmassenhove et al., 2018).

Gender tagging is effective: a Sequence-toSequence Neural Network trained on Europarl increased BLEU scores significantly for machine translations from English to French in which the first-person speaker was female (Vanmassenhove et al., 2018). Sentences with male first-person speakers had accuracy increases by a sizeable margin. However, gender-tagging can be expensive: knowing the gender of the source of a data point requires meta-information, and obtaining this could be costly in terms of memory usage and time. Furthermore, MT models may need to be redesigned to correctly parse the gender tags.

\subsubsection{Bias Fine-Tuning}

Unbiased data sets for a given task may be scarce, but there may exist unbiased data sets for a related task. Bias fine-tuning incorporates transfer learning from an unbiased data set to ensure that a model contains minimal bias before fine-tuning the model on a more biased data set used to train for the target task directly (Park et al., 2018). This allows models to avoid learning biases from training sets while still being adequately trained to perform a task.

Bias fine-tuning has been shown to be relatively effective. Park et al. (2018) use transfer learning from a gender unbiased abusive tweets data set (Founta et al., 2018) and fine-tuning on a genderbiased sexist tweets data set (Waseem and Hovy, 2016) to train a Convolutional Neural Network (CNN). They evaluate the CNN using a GBET evaluation set (which is private, so not mentioned in 2.3). They tested the same model after training it on gender-swapped data sets as well. Park et al. (2018) find that gender-swapping was more effective at both removing bias and retaining performance than bias fine-tuning. However, transfer learning may have been ineffective in this case because abusive language detection data sets and sexist language detection data sets have significant differences. For more similar data sets, bias finetuning may be more effective; further testing is necessary.

\subsection{Debiasing Gender in Word Embeddings}

Word embeddings represent words in a vector space. These embeddings have been demonstrated to reflect societal biases and changing views during social movements in the United States (Garg et al., 2018).

As the word embedding model is a fundamental component in many NLP systems, mitigating bias in embeddings plays a key role in the reduction of bias that is propagated to downstream tasks (e.g., (Zhao et al., 2018a)). However, it is debatable if debiasing word embeddings is a philosophically right step towards mitigating bias in NLP. Caliskan et al. (2017) argue that debiasing word embeddings blinds an AI agent's perception rather than teaching it to perform fair actions. We refer readers to the discussion in (Caliskan et al., 2017).

It is also important to recognize that removing gender bias from the embedding space entirely is difficult. While existing methods successfully mitigate bias with respect to projection onto the gender subspace in some degrees, Gonen and Goldberg (2019) show that gender bias based on more subtle metrics such as cluster bias still exist.

In the following we review two families of approaches to debias gender in word embeddings. One difference between these two types of methods is that the former does not require retraining embeddings, whereas the latter does.

\subsubsection{Removing Gender Subspace in Word Embeddings}

Schmidt (2015) first removed similarity to the gender subspace in word embeddings by building a genderless framework using cosine similarity and orthogonal vectors (Schmidt, 2015). Removing the gender component, though, pushes the word he to become the 6th closest word to she when it was the 1,826 th closest previously. The genderless 


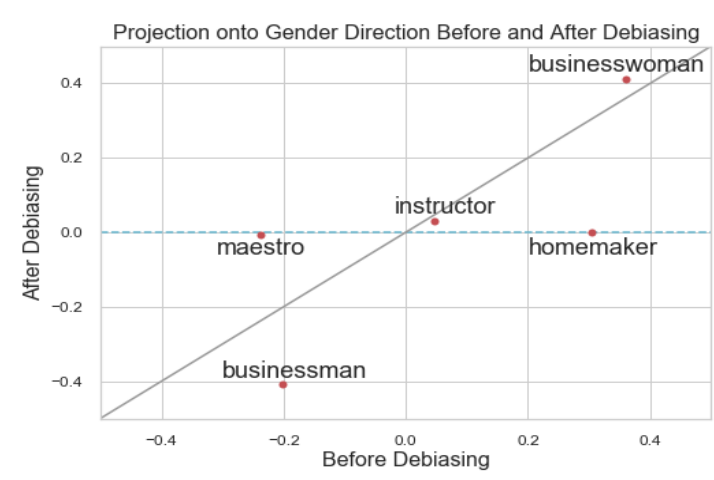

Figure 2: We project five word2vec embeddings onto the 'he' - 'she' direction before and after neutralizing the gender-neutral words maestro, instructor, and homemaker and equalizing the gender-specific pair businessman and businesswoman (Bolukbasi et al., 2018). For both $x$ and $y$-axes, negative values represent male gender bias and positive values represent female gender bias.

framework may be flawed because the semantic definition of a given word may be closely tied to its gender component. However, a case can also be made that a word's gender component should play a key role in its semantic definition. We encourage future work to collaborate with social scientists for further discussion on this topic.

Bolukbasi et al. (2016) build upon Schmidt (2015) and propose to surgerically alter the embedding space by removing the gender component only from gender-neutral words. Instead of removing gender altogether, debiasing involves making gender-neutral words orthogonal to the gender direction (see Figure 2). Ultimately, word embeddings with reduced bias performed just as well as unaltered embeddings on coherence and analogy-solving tasks (Bolukbasi et al., 2016).

\subsubsection{Learning Gender-Neutral Word Embeddings}

Zhao et al. (2018b) propose a new method called GN-GloVe that does not use a classifier to create a set of gender-specific words. The authors train the word embeddings by isolating gender information in specific dimensions and maintaining gender-neutral information in the other dimensions. They do this by (1) minimizing the negative difference (i.e. maximizing the difference) between the gender dimension in male and female definitional word embeddings and (2) maximizing the difference between the gender direction and the other neutral dimensions in the word embed- dings. This allows for greater flexibility; the gender dimensions can be used or neglected.

Finally, we note that both aforementioned approaches (Bolukbasi et al., 2016; Zhao et al., 2018b) used to debias word embeddings may not work with embeddings in a non-Euclidean space, such as Poincare embeddings (Nickel and Kiela, 2017), because the notion of cosine similarity would no longer apply. Also, it is unclear if these approaches can be extended to other languages beyond English, especially for languages with grammatical genders.

\section{Debiasing by Adjusting Algorithms}

Some gender debiasing methods in NLP adjust predictions in NLP systems. We call these algorithm adjustment methods. In this section, we discuss two such approaches.

\subsection{Constraining Predictions}

Zhao et al. (2017) show that an NLP model risks amplifying bias by making predictions which exacerbate biases present in the training set. For instance, if $80 \%$ of coreferents of "secretary" are female in a training set and a model trained on that set predicts $90 \%$ of coreferents of "secretary" in a test set to be female, then that model amplifies bias.

Zhao et al. (2017) proposed Reducing Bias Amplification (RBA) based on a constrained conditional model (Roth and Yih, 2004), which takes an existing model's optimization function and constrains that function to ensure its predictions fit defined conditions. For example, when RBA was applied to the visual semantic role labelling (Yatskar et al., 2016), it restricted the ratio of males to females predicted to be doing particular activities to prevent the model from amplifying bias through predictions. The approximate inference can be efficient solved by Lagrangian relaxation (Rush and Collins, 2012).

\subsection{Adversarial Learning: Adjusting the Discriminator}

Zhang et al. (2018) propose a variation on the traditional generative adversarial network (Goodfellow et al., 2014) by having the generator learn with respect to a protected gender attribute. In other words, the generator attempts to prevent the discriminator from identifying the gender in a given task such as analogy completion. This method has 
the potential to be generalizable: it can be used to debias any model that uses gradient-based learning.

\section{Conclusion and Future Directions}

In this paper, we summarize recent literature about recognizing and mitigating gender bias in NLP. We acknowledge that the scope of this paper is limited. There is a long history of gender stereotype study in law, psychology, media study, and many other disciplines which we do not discuss. Similar issues of algorithmic bias have also been discussed extensively in artificial intelligence, machine learning, data mining, and several other application fields (e.g., (Calders and Verwer, 2010; Feldman et al., 2015; Hardt et al., 2016; Misra et al., 2016; Kleinberg et al., 2016; Pleiss et al., 2017; Beutel et al., 2017; Misra et al., 2016)). Other important aspects such as model/data transparency (Mitchell et al., 2019; Bender and Friedman, 2018) and privacy preservation (Reddy and Knight, 2016; Elazar and Goldberg, 2018; Li et al., 2018) are also not covered in this literature survey. Besides, we refer the readers to Hovy and Spruit (2016) for a more general discussion of ethical concern in NLP.

The study of gender bias in NLP is still relatively nascent and consequently lacks unified metrics and benchmarks for evaluation. We urge researchers in related fields to work together to create standardized metrics that rigorously measure the gender bias in NLP applications. However, we recognize that different applications may require different metrics and there are trade-offs between different notions of biases (Barocas et al., 2018; Chouldechova and Roth, 2018).

Gender debiasing methods in NLP are not sufficient to debias models end-to-end for many applications. We note the following limitations of current approaches. First, the majority of debiasing techniques focus on a single, modular process of an end-to-end NLP system. It remains to be discovered how these individual parts harmonize together to form an ideally unbiased system. Second, most gender debiasing methods have only been empirically verified in limited applications (Zhang et al., 2018; Zhao et al., 2017), and it is not clear that these methods can generalize to other tasks or models. Third, we note that certain debiasing techniques may introduce noise into a NLP model, causing performance degradation. Finally, hand-craft debiasing approaches may unintentionally encode the implicit bias of the developers.

Below, we identify a few future directions.

Mitigating Gender Bias in Languages Beyond English. With few exceptions (Vanmassenhove et al., 2018; Prates et al., 2018), prior work has focused on mitigating gender bias in the English language. Future work can look to apply existing methods or devise new techniques towards mitigating gender bias in other languages as well. However, such a task is not trivial. Methods such as gender-swapping are relatively easy in English because English does not distinguish gender linguistically. However, in languages such as Spanish, each noun has its own gender and corresponding modifiers of the noun need to align with the gender of the noun. To perform gender-swapping in such languages, besides swapping those gendered nouns, we also need to change the modifiers.

Non-Binary Gender Bias. With few exceptions (Manzini et al., 2019), work on debiasing in NLP has assumed that the protected attribute being discriminated against is binary. Non-binary genders (Richards et al., 2016) as well as racial biases have largely been ignored in NLP and should be considered in future work.

Interdisciplinary Collaboration. As mentioned in Section 1, gender bias is not a problem that is unique to NLP; other fields in computer science such as data mining, machine learning, and security also study gender bias (Calders and Verwer, 2010; Feldman et al., 2015; Hardt et al., 2016; Misra et al., 2016; Kleinberg et al., 2016; Pleiss et al., 2017; Beutel et al., 2017; Kilbertus et al., 2017). Many of these technical methods could be applicable to NLP yet to our knowledge have not been studied.

Additionally, mitigating gender bias in NLP is both a sociological and an engineering problem. To completely debias effectively, it is important to understand how machine learning methods encode biases and how humans perceive biases. A few interdisciplinary studies (Herbelot et al., 2012; Avin et al., 2015; Fu et al., 2016; Schluter, 2018) have emerged, and we urge more interdisciplinary discussions in terms of gender bias. Approaches from other technical fields may improve current debiasing methods in NLP or inspire the development of new, more effective methods even if the properties of the data or 
problem are different across fields. Discussions between computer scientists and sociologists may improve understanding of latent gender bias found in machine learning data sets and model predictions.

\section{Acknowledgements}

We thank anonymous reviewers for their helpful feedback. We also acknowledge the thoughtful talks in related topics by Kate Crawford, Margaret Mitchell, Joanna J. Bryson, and several others. This material is based upon work supported in part by the National Science Foundation under Grants 1821415 and 1760523.

\section{References}

Chen Avin, Barbara Keller, Zvi Lotker, Claire Mathieu, David Peleg, and Yvonne-Anne Pignolet. 2015. Homophily and the Glass Ceiling Effect in Social Networks. In Proceedings of the 2015 Conference on Innovations in Theoretical Computer Science (ITCS'15), pages 41-50. ACM.

Solon Barocas, Moritz Hardt, and Arvind Narayanan. 2018. Fairness and Machine Learning. fairmlbook.org. http: / / www. fairmlbook.org.

Emily M Bender and Batya Friedman. 2018. Data statements for natural language processing: Toward mitigating system bias and enabling better science. Transactions of the Association for Computational Linguistics, 6:587-604.

Alex Beutel, Jilin Chen, Zhe Zhao, and Ed Huai hsin Chi. 2017. Data Decisions and Theoretical Implications when Adversarially Learning Fair Representations. In 2017 Workshop on Fairness, Accountability, and Transparency in Machine Learning.

Tolga Bolukbasi, Kai-Wei Chang, James Y Zou, Venkatesh Saligrama, and Adam T Kalai. 2016. Man Is to Computer Programmer As Woman Is to Homemaker? Debiasing Word Embeddings. In Neural Information Processing Systems (NIPS ‘16).

Tolga Bolukbasi, Kai-Wei Chang, James Y Zou, Venkatesh Saligrama, and Adam T Kalai. 2018. Debiaswe. https://bit.ly/2ruopBz. Accessed on 12.10.2018.

Kaylee Burns, Lisa Anne Hendricks, Trevor Darrell, Anna Rohrbach, and Kate Saenko. 2018. Women Also Snowboard: Overcoming Bias in Captioning Models. European Conference on Computer Vision (EECV'18).

Toon Calders and Sicco Verwer. 2010. Three Naive Bayes Approaches for Discrimination-Free Classification. Data Mining and Knowledge Discovery, 21(2):277-292.
Aylin Caliskan, Joanna J Bryson, and Arvind Narayanan. 2017. Semantics Derived Automatically from Language Corpora Contain Human-Like Biases. Science, 356(6334):183-186.

Alexandra Chouldechova and Aaron Roth. 2018. The Frontiers of Fairness in Machine Learning. arXiv preprint arXiv:1810.08810.

Kate Crawford. 2017. The Trouble With Bias. Keynote at Neural Information Processing Systems (NIPS‘17).

Lucas Dixon, John Li, Jeffrey Sorensen, Nithum Thain, and Lucy Vasserman. 2017. Measuring and Mitigating Unintended Bias in Text Classification. In Association for the Advancement of Artificial Intelligence (AAAI'17).

Laura Douglas. 2017. AI is not Just Learning our Biases; It Is Amplifying Them. https : / / bit. Iy / 2 zRvGhH. Accessed on 11.15.2018.

Yanai Elazar and Yoav Goldberg. 2018. Adversarial Removal of Demographic Attributes from Text Data. In Empirical Methods of Natural Language Processing (EMNLP'18).

Michael Feldman, Sorelle A Friedler, John Moeller, Carlos Scheidegger, and Suresh Venkatasubramanian. 2015. Certifying and Removing Disparate Impact. In Proceedings of the 21th ACM SIGKDD International Conference on Knowledge Discovery and Data Mining (KDD`15).

Antigoni-Maria Founta, Constantinos Djouvas, Despoina Chatzakou, Ilias Leontiadis, Jeremy Blackburn, Gianluca Stringhini, Athena Vakali, Michael Sirivianos, and Nicolas Kourtellis. 2018. Large Scale Crowdsourcing and Characterization of Twitter Abusive Behavior. In Association for the Advancement of Artifical Intelligence (AAAI'18).

Liye Fu, Cristian Danescu-Niculescu-Mizil, and Lillian Lee. 2016. Tie-breaker: Using Language Models to Quantify Gender Bias in Sports Journalism. In Proceedings of the IJCAI workshop on NLP meets Journalism.

Nikhil Garg, Londa Schiebinger, Dan Jurafsky, and James Zou. 2018. Word Embeddings Quantify 100 Years of Gender and Ethnic Stereotypes. Proceedings of the National Academy of Sciences, 115(16):E3635-E3644.

Hila Gonen and Yoav Goldberg. 2019. Lipstick on a Pig: Debiasing Methods Cover up Systematic Gender Biases in Word Embeddings But Do Not Remove Them. In North American Chapter of the Association for Computational Linguistics (NAACL'19).

Ian Goodfellow, Jean Pouget-Abadie, Mehdi Mirza, Bing Xu, David Warde-Farley, Sherjil Ozair, Aaron Courville, and Yoshua Bengio. 2014. Generative Adversarial Nets. In Advances in Neural Information Processing Systems (NIPS'14). 
Anthony G Greenwald, Debbie E McGhee, and Jordan LK Schwartz. 1998. Measuring Individual Differences in Implicit Cognition: The Implicit Association Test. Journal of Personality and Social Psychology, 74(6):1464.

Moritz Hardt, Eric Price, and Nati Srebro. 2016. Equality of Opportunity in Supervised Learning. In Advances in Neural Information Processing Systems (NIPS'16).

Tatsunori B Hashimoto, Megha Srivastava, Hongseok Namkoong, and Percy Liang. 2018. Fairness Without Demographics in Repeated Loss Minimization.

Aurélie Herbelot, Eva Von Redecker, and Johanna Müller. 2012. Distributional Techniques for Philosophical Enquiry. In Proceedings of the 6th Workshop on Language Technology for Cultural Heritage, Social Sciences, and Humanities, pages 4554. Association for Computational Linguistics.

Dirk Hovy and Shannon L Spruit. 2016. The Social Impact of Natural Language Processing. In Proceedings of the 54th Annual Meeting of the Association for Computational Linguistics (ACL'16), volume 2, pages 591-598.

Niki Kilbertus, Mateo Rojas-Carulla, Giambattista Parascandolo, Moritz Hardt, Dominik Janzing, and Bernhard Schölkopf. 2017. Avoiding Discrimination Through Causal Reasoning. In Neural Information Processing Systems (NIPS'17).

Svetlana Kiritchenko and Saif M Mohammad. 2018. Examining Gender and Race Bias in Two Hundred Sentiment Analysis Systems. In 7th Joint Conference on Lexical and Computational Semantics (SEM'18).

Jon Kleinberg, Sendhil Mullainathan, and Manish Raghavan. 2016. Inherent Trade-offs in the Fair Determination of Risk Scores. In Computing Research Repository (CoRR '16).

Kenton Lee, Luheng He, Mike Lewis, and Luke Zettlemoyer. 2017. End-to-End Neural Coreference Resolution. In Empirical Methods of Natural Language Processing (EMNLP'17).

Kenton Lee, Luheng He, and Luke Zettlemoyer. 2018. Higher-Order Coreference Resolution with Coarseto-Fine Inference. In Empirical Methods of Natural Language Processing (EMNLP'18).

Yitong Li, Timothy Baldwin, and Trevor Cohn. 2018. Towards Robust and Privacy-Preserving Text Representations. In Proceedings of the 56th Annual Meeting of the Association for Computational Linguistics (ACL'18), pages 1650-1659.

Kaiji Lu, Piotr Mardziel, Fangjing Wu, Preetam Amancharla, and Anupam Datta. 2018. Gender Bias in Neural Natural Language Processing.
Nishtha Madaan, Sameep Mehta, Taneea Agrawaal, Vrinda Malhotra, Aditi Aggarwal, Yatin Gupta, and Mayank Saxena. 2018. Analyze, Detect and Remove Gender Stereotyping from Bollywood Movies. In Conference on Fairness, Accountability and Transparency (FAT'18), pages 92-105.

Thomas Manzini, Yao Chong Lim, Yulia Tsvetkov, and Alan W Black. 2019. Black is to Criminal as Caucasian is to Police: Detecting and Removing Multiclass Bias in Word Embeddings. In North American Chapter of the Association for Computational Linguistics (NAACL'19).

Chandler May, Alex Wang, Shikha Bordia, Samuel R Bowman, and Rachel Rudinger. 2019. On Measuring Social Biases in Sentence Encoders. In North American Chapter of the Association for Computational Linguistics (NAACL'19).

Ishan Misra, C. Lawrence Zitnick, Margaret Mitchell, and Ross Girshick. 2016. Seeing Through the Human Reporting Bias: Visual Classifiers from Noisy Human-Centric Labels. In Proceedings of the IEEE Conference on Computer Vision and Pattern Recognition (IEEE'16), pages 2930-2939.

Margaret Mitchell, Simone Wu, Andrew Zaldivar, Parker Barnes, Lucy Vasserman, Ben Hutchinson, Elena Spitzer, Inioluwa Deborah Raji, and Timnit Gebru. 2019. Model cards for model reporting. In Proceedings of the Conference on Fairness, Accountability, and Transparency, pages 220-229. ACM.

Corinne A Moss-Racusin, John F Dovidio, Victoria L Brescoll, Mark J Graham, and Jo Handelsman. 2012. Science Faculty's Subtle Gender Biases Favor Male Students. Proceedings of the National Academy of Sciences, 109(41):16474-16479.

Maximillian Nickel and Douwe Kiela. 2017. Poincarè Embeddings for Learning Hierarchical Representations. In Advances in Neural Information Processing Systems (NIPS`17), pages 6338-6347.

Brian A Nosek, Frederick L Smyth, Natarajan Sriram, Nicole M Lindner, Thierry Devos, Alfonso Ayala, Yoav Bar-Anan, Robin Bergh, Huajian Cai, Karen Gonsalkorale, Selin Kesebir, Norbert Maliszewski, Felix Neto, Eero Olli, Jaihyun Park, Konrad Schnabel, Kimihiro Shiomura, Bogdan Tulbure, Reinout Wiers, Monika Somogyi, Nazar Akrami, Bo Ekehammar, Michelangelo Vianello, Mahzarin Banaji, and Anthony Greenwald. 2009. National Differences in Gender-Science Stereotypes Predict National Sex Differences in Science and Math Achievement. Proceedings of the National Academy of Sciences, 106(26):10593-10597.

Ji Ho Park, Jamin Shin, and Pascale Fung. 2018. Reducing Gender Bias in Abusive Language Detection. In Empirical Methods of Natural Language Processing (EMNLP'18). 
Matthew E Peters, Mark Neumann, Mohit Iyyer, Matt Gardner, Christopher Clark, Kenton Lee, and Luke Zettlemoyer. 2018. Deep Contextualized Word Representations. In North American Chapter of the Association for Computational Linguistics (NAACL'18).

Geoff Pleiss, Manish Raghavan, Felix Wu, Jon Kleinberg, and Kilian Q Weinberger. 2017. On Fairness and Calibration. In Advances in Neural Information Processing Systems (NIPS`17), pages 5680-5689.

Marcelo O. R. Prates, Pedro H. Avelar, and Luís C. Lamb. 2018. Assessing gender bias in machine translation: a case study with Google Translate. Neural Computing and Applications.

Sravana Reddy and Kevin Knight. 2016. Obfuscating Gender in Social Media Writing. In Proceedings of the First Workshop on NLP and Computational Social Science, pages 17-26.

Christina Richards, Walter Pierre Bouman, Leighton Seal, Meg John Barker, Timo O Nieder, and Guy $\mathrm{T}$ 'Sjoen. 2016. Non-Binary or Genderqueer Genders. International Review of Psychiatry (IRP'16“).

Dan Roth and Wen-tau Yih. 2004. A linear programming formulation for global inference in natural language tasks. In Proceedings of the Eighth Conference on Computational Natural Language Learning (CoNLL-2004) at HLT-NAACL 2004.

Rachel Rudinger, Jason Naradowsky, Brian Leonard, and Benjamin Van Durme. 2018. Gender Bias in Coreference Resolution. In North American Chapter of the Association for Computational Linguistics (NAACL'18).

Alexander M Rush and Michael Collins. 2012. A Tutorial on Dual Decomposition and Lagrangian Relaxation for Inference in Natural Language Processing. Journal of Artificial Intelligence Research, 45:305362.

Natalie Schluter. 2018. The Glass Ceiling in NLP. In Empirical Methods of Natural Language Processing (EMNLP'18).

Ben Schmidt. 2015. Rejecting the Gender Binary: A Vector-Space Operation. https://bit.ly/ 1OhXJM0. Accessed on 11.15.2018.

Rachel Tatman. 2017. Gender and Dialect Bias in YouTube's Automatic Captions. In Proceedings of the First ACL Workshop on Ethics in Natural Language Processing (ACL'17), pages 53-59.

Eva Vanmassenhove, Christian Hardmeier, and Andy Way. 2018. Getting Gender Right in Neural Machine Translation. In Empirical Methods of Natural Language Processing (EMNLP'18).

Zeerak Waseem and Dirk Hovy. 2016. Hateful Symbols or Hateful People? Predictive Features for Hate Speech Detection on Twitter. In North American
Chapter of the Association for Computational Linguistics (NAACL'16).

Kellie Webster, Marta Recasens, Vera Axelrod, and Jason Baldridge. 2018. Mind the GAP: A Balanced Corpus of Gendered Ambiguous Pronouns. In Transactions of the ACL (TACL'18).

Mark Yatskar, Luke Zettlemoyer, and Ali Farhadi. 2016. Situation Recognition: Visual Semantic Role Labeling for Image Understanding. In Proceedings of the IEEE Conference on Computer Vision and Pattern Recognition (IEEE '16), pages 5534-5542.

Brian $\mathrm{Hu}$ Zhang, Blake Lemoine, and Margaret Mitchell. 2018. Mitigating Unwanted Biases with Adversarial Learning. In AAAI/ACM Conference on Artificial Intelligence, Ethics, and Society (AIES'18).

Jieyu Zhao, Tianlu Wang, Mark Yatskar, Ryan Cotterell, Vicente Ordonez, and Kai-Wei Chang. 2019. Gender Bias in Contextualized Word Embeddings.

Jieyu Zhao, Tianlu Wang, Mark Yatskar, Vicente Ordonez, and Kai-Wei Chang. 2017. Men Also Like Shopping: Reducing Gender Bias Amplification Using Corpus-Level Constraints. In Empirical Methods of Natural Language Processing (EMNLP'17).

Jieyu Zhao, Tianlu Wang, Mark Yatskar, Vicente Ordonez, and Kai-Wei Chang. 2018a. Gender Bias in Coreference Resolution: Evaluation and Debiasing Methods. In North American Chapter of the Association for Computational Linguistics (NAACL'18).

Jieyu Zhao, Yichao Zhou, Zeyu Li, Wei Wang, and KaiWei Chang. 2018b. Learning Gender-Neutral Word Embeddings. In Empirical Methods of Natural Language Processing (EMNLP'18). 